\title{
Seismological Studies for Tensile Faults
}

\author{
Gwo-Bin $\mathrm{Ou}^{*}$ \\ Institute of Seismology, National Chung Cheng University, Chia-Yi 621, Taiwan, ROC
}

Received 13 June 2007, accepted 22 October 2007

\begin{abstract}
A shear slip fault, an equivalence of a double couple source, has often been assumed to be a kinematic source model in ground motion simulation. Estimation of seismic moment based on the shear slip model indicates the size of an earthquake. However, if the dislocation of the hanging wall relative to the footwall includes not only a shear slip tangent to the fault plane but also expansion and compression normal to the fault plane, the radiating seismic waves will feature differences from those out of the shear slip fault. Taking account of the effects resulting from expansion and compression to a fault plane, we can resolve the tension and pressure axes as well as the fault plane solution more exactly from ground motions than previously, and can evaluate how far a fault zone opens or contracts during a developing rupture. In addition to a tensile angle and Poisson's ratio for the medium, a tensile fault with five degrees of freedom has been extended from the shear slip fault with only three degrees of freedom, strike, dip, and slip.
\end{abstract}

Key words: Tensile fault, Moment tensor, Radiation pattern, Focal mechanism

Citation: Ou, G. B., 2008: Seismological studies for tensile faults. Terr. Atmos. Ocean. Sci., 19, 463-471, doi: 10.3319/TAO.2008.19.5.463(T)

\section{INTRODUCTION}

The shear slip model has played an important role in earthquake source studies because historical earthquakes present on-fault offsets of rock breaking. The final slip in the fault plane has always been assumed to be the result of a seismic source without nets of force, torque, and volume change. The radiations of $P$ and $S$ waves for shear slip faults are concluded with remarkably straight-forward formulas (Aki and Richards 1980; Herrmann 1989; Pujol and Herrmann 1990). In addition to shear faulting, a possible expansive and/or compressive process in a direction normal to the fault plane during rupture has been reported and proposed. The historical record of an M7 earthquake at Chia-Yi, Taiwan, in 1792 tells of a ravine trail cracking and suddenly opening. At which point a woodsman fell into it, then the earth quickly shut again (Xie and Cai 1987). Haskell (1964) found that $P: S$ amplitude ratios at high frequencies are usually larger than those expected for shear faulting, and suggested that this indicates fault normal motion caused by fault roughness. Miller et al. (1998) concluded that shallow earthquakes unlike their planar idealizations have real fault surfaces which are rough, so that shear slip must involve

\footnotetext{
* Corresponding author

E-mail: seigbo@eq.ccu.edu.tw
}

some motion normal to fault segments. Scholz (1990) assumed that volume dilatancy occurs within a fault zone as slip occurs during nucleation prior to a macroscopic shear crack. The fault zone dilatancy mechanism may be either joint dilatancy, in which the fault walls must move apart to accommodate slip, or dilatancy due to shear of granular materials, such as gouge or breccia, within a fault zone. If these expansive cracks twist into a compressive shear faulting, the fault zone will finally undergo a closing of voids or volume compaction. Julian et al. (1998) pointed out that compressive stress tends to prevent voids from forming at depth in the Earth, but high fluid pressure in volcanic and geothermal areas can overcome this effect and allow opening tensile failure to occur.

In seismological studies, the displacement field outside the earthquake source region is simulated by that produced in an unfaulted medium over the fault surface by a distribution of pairs of force couples. The force system is represented by a moment tensor (Gilbert 1970), which is divided into isotropic and deviatoric parts. It is common in seismological research to decompose the deviatoric part further into a linear combination of a double couple (DC) and a compensated linear vector dipole (CLVD) (Knopoff and Randall 1970; Julian et al. 1998). The method used in the Harvard 
centroid moment tensor gives the largest possible DC that has a CLVD remainder (Dziewonski et al. 1987). Well constrained non-DC earthquakes have been observed in many environments, including volcanic and geothermal areas, mines, and deep subduction zones (Frohlich 1994; Miller et al. 1998; Templeton and Dreger 2006). Since the decomposition of the deviatoric moment tensor is nonunique (Jost and Herrmann 1989; Frohlich 1994), then decomposition for the definition of deviatoric moment is more troublesome. On the other hand, the isotropic part definitely explains the volume change of seismic source (Müller 2001). An $\mathrm{M}_{\mathrm{L}} 6.8$ Hualien, Taiwan, earthquake occurred at a depth of $15 \mathrm{~km}$ on 14 November 1986. Zheng et al. (1995) inverted $P$ and $\mathrm{SH}$ waveforms and yielded a moment tensor equivalent to a reverse slip DC combined with an implosion. An M 4.6 earthquake on 10 February 1987 beneath the Kanto district, Japan, had dilatational $P$ wave polarities over most of the focal sphere (Hurukawa and Imoto 1993). The polarities are consistent with conic nodal surfaces with an apex angle of about $78^{\circ}$, implying an implosive isotropic component. Dreger et al. (2000) found that dilatational processes accompanied four earthquakes within a few days and a few kilometers of one another in 1997 in the Long Valley Caldera with isotropic moments of $27-42 \%$ of the deviatoric moments. Panza and Sarao (2000) suggested the isotropic components of moment tensors can be found in volcanic and geothermal areas.

A symmetric moment tensor for an earthquake involving isotropic and deviatoric parts can be a combination of opening and/or closing and shear faulting. The opening facilitates slippage and decreases the amount of heat generated (Anooshehpoor and Brune 1992, 1994). It might resolve some paradoxes about faulting, such as how faults can slip under extremely low driving shear stresses (Zoback et al. 1987) and why friction does not produce large heat flow anomalies near major faults (Lachenbruch and Sass 1980). The opening is transient, with the fault surface closed both before and after an earthquake. This phenomenon will not contribute to moment tensors of temporal order zero, only explicitly time-dependent or higher-order temporal moments can represent transient opening (Julian et al. 1998). He et al. (2003) have extended this idea to include opening tensile point sources from a point pure shear dislocation source to examine surface static displacements.

Faults with shear slip as well as expansion and/or compression will be called tensile faults in this article. An arbitrary jump of displacement on a tensile fault segment can be equivalent to a distribution of linear dipoles and double couples having moments at the source (Burridge and Knopoff 1964; Aki and Richards 1980; Ben-Menahem and Singh 1981; Kennett 1983; Kostrov and Das 1988). In this study, we will express and discuss the moment tensor, $P$ and $S$ wave motions, and their radiation patterns in the case of tensile faults. The pressure and tension axes, the tensile angle, and the fault plane solution are also analyzed from its moment tensor.

\section{MOMENT TENSOR}

Dislocation theory for an earthquake source assumes that a jump in the displacement vector across an internal surface $\sum$ occurs inside the Earth. The displacement vector can be tangential to the fault surface or normal to it. We define the displacement vector $\Delta \mathbf{u}$ as the movement of the hanging wall surface $\Sigma^{+}$relative to the footwall surface $\Sigma^{-}$and the unit normal vector $\hat{\mathbf{n}}$ of the fault is directed from $\Sigma^{-}$to $\Sigma^{+}$. Ground motions resulting from such a dislocation on a limited fault segment can be simulated from a set of nine couples in an unfaulted medium with forces and arms in any of three mutually perpendicular directions. As it stands, a tensile fault can correspond to many representations of nine couples, so the choice of nine couples is not unique. Ground motions radiated from any set of nine couples corresponding to the same dislocation will be equal. The moments $M_{l m}(t)$ of nine couples with forces and arms in $l$ and $m$ directions for a tensile fault segment normal to $\hat{\mathbf{n}}$ with a displacement vector function $\Delta \mathbf{u}(t)$ of time $t$ in an isotropic medium are given by:

$M_{l m}(t)=A\left\{\lambda \delta_{l m} \Delta u_{i}(t) n_{i}+\mu\left[\Delta u_{l}(t) n_{m}+\Delta u_{m}(t) n_{l}\right]\right\}$

(Aki and Richards 1980). The summation convention over repeated indices is used. $A$ is the area of the fault segment. $\lambda$ and $\mu$ are the Lamé constants of the medium, where $\mu$ is shear modulus. $\delta_{l m}$ is the Kronecker delta, which is equal to 1 if $l=m$ and 0 otherwise. For an example, $M_{l 1}$ represents the force couple with arm in the 1-direction. Projecting the forces of the couple in the 1-direction, we get a linear dipole having a moment of $M_{11}$. Two force projections in the 2- and 3-directions are two shear couples having moments of $M_{21}$ and $M_{31}$. Nine elements of $M_{l m}$ constitute a moment tensor $\mathbf{M}$ for a tensile fault segment. The moment tensor $\mathbf{M}$ is a symmetric matrix. The diagonal elements of $\mathbf{M}$ are moments of the linear dipoles along three mutually perpendicular directions, which we choose. Each of the linear dipoles has zero net force and zero net torque. The off-diagonal elements $M_{l m}$ and $M_{m l}, l \neq m$, are equal and express the moments of a double couple, which also produce zero net force and zero net torque.

Let the displacement vector function $\Delta \mathbf{u}(t)$ of a tensile fault segment be decomposed into a shear component tangent to the fault and a tensile component normal to it (Kostrov and Das 1988):

$\Delta \mathbf{u}(t)=\Delta u_{(n)}(t) \hat{\mathbf{n}}+\Delta u_{(f)}(t) \hat{\mathbf{f}}$

where $\Delta u_{(n)}(t)$ and $\Delta u_{(f)}(t)$ are the tensile function and the 
shear function of a fault segment; and $\hat{\mathbf{f}}$ is the unit slip vector in the fault plane. Then the moment tensor function formed by a linear combination of $\Delta u_{(n)}(t)$ and $\Delta u_{(f)}(t)$ for a tensile fault element is:

$$
\begin{aligned}
M_{l m}(t) & =A\left[\left(\lambda \delta_{l m}+2 \mu n_{l} n_{m}\right) \Delta u_{(n)}(t)\right. \\
& \left.+\mu\left(f_{l} n_{m}+f_{m} n_{l}\right) \Delta u_{(f)}(t)\right]
\end{aligned}
$$

$\Delta u_{(n)}(t)$ is positive when the fault segment expands and negative when it compresses. The first term in the bracket on the right hand side of Eq. (3) comes from a tensile motion, expansion and compression. The second term, which is a double couple source, results from shear faulting. If there is no net tensile motion at the end of faulting, the final moment tensor of the fault segment will be:

$M_{l m}=M_{0}\left(f_{l} n_{m}+f_{m} n_{l}\right)$

where $M_{0}$ defined as $\mu A u_{d}$ is the seismic moment of the fault segment, $u_{d}$ is the final shear slip in the fault plane. The seismic moment $M_{0}$ is a static source parameter for shear faulting.

Let us consider the moment tensor in three mutually perpendicular directions, the first is along the slip vector $\hat{\mathbf{f}}$, the second along the fault normal vector $\hat{\mathbf{n}}$, and the third along $\hat{\mathbf{f}} \times \hat{\mathbf{n}}$. In case of shear faulting, $\Delta u_{(n)}(t)=0$, only the elements $M_{12}(t)$ and $M_{21}(t)$ will be nonzero. The moment tensor function of a shear component is:

$\mathbf{M}_{(f)}(t)=A \Delta u_{(f)}(t)\left[\begin{array}{ccc}0 & \mu & 0 \\ \mu & 0 & 0 \\ 0 & 0 & 0\end{array}\right]$

The shear faulting is equivalent to a double couple, having a moment function of $\mu A \Delta u_{(f)}(t)$, in the plane containing $\hat{\mathbf{f}}$ and $\hat{\mathbf{n}}$. In case of tensile motion, $\Delta u_{(f)}(t)=0$, the non-diagonal elements of tensor vanish. The moment tensor function of a tensile component can be expressed by:

$\mathbf{M}_{(n)}(t)=A \Delta u_{(n)}(t)\left\{\left[\begin{array}{ccc}\lambda & 0 & 0 \\ 0 & \lambda & 0 \\ 0 & 0 & \lambda\end{array}\right]+\left[\begin{array}{ccc}0 & 0 & 0 \\ 0 & 2 \mu & 0 \\ 0 & 0 & 0\end{array}\right]\right\}$

The tensile motion is equivalent to a combination of an isotropic source having a moment function of $\lambda A \Delta u_{(n)}(t)$ and a linear dipole having a moment function of $2 \mu A \Delta u_{(n)}(t)$ along $\hat{\mathbf{n}}$. It is clear that the moment tensor of a tensile motion includes a function of volume change. The trace of moment tensor gives the volume change $A \Delta u_{(n)}(t)$, which is $\operatorname{tr}[\mathbf{M}(t)] /(3 \lambda+2 \mu)$ (Müller 2001).
For a tensile fault segment we define fault parameters as shown in Fig. 1. The strike $\phi_{s}$ of a fault is the angle measured clockwise from north such that the fault plane dips down to the right when looking in the direction of strike. The $\operatorname{dip} \delta$ is the angle of the fault plane measured from the horizontal in a downward direction. The $\operatorname{slip} \lambda_{s}$ is the angle of motion of the hanging wall relative to the footwall measured in the fault plane from the direction of strike in a counterclockwise sense. In Cartesian coordinates of north $\left(x_{1}\right)$, east $\left(x_{2}\right)$, and down $\left(x_{3}\right)$, the unit slip vector $\hat{\mathbf{f}}$ and the unit fault normal $\hat{\mathbf{n}}$ expressed by fault parameters $\left(\phi_{s}, \delta, \lambda_{s}\right)$ are:

$$
\begin{aligned}
\hat{\mathbf{f}} & =\left(\cos \lambda_{s} \cos \phi_{s}+\cos \delta \sin \lambda_{s} \sin \phi_{s}\right) \hat{\mathbf{x}}_{1} \\
& +\left(\cos \lambda_{s} \sin \phi_{s}-\cos \delta \sin \lambda_{s} \cos \phi_{s}\right) \hat{\mathbf{x}}_{2}-\sin \delta \sin \lambda_{s} \hat{\mathbf{x}}_{\mathbf{3}} \\
\hat{\mathbf{n}} & =-\sin \delta \sin \phi_{s} \hat{\mathbf{x}}_{1}+\sin \delta \cos \phi_{s} \hat{\mathbf{x}}_{\mathbf{2}}-\cos \delta \hat{\mathbf{x}}_{3}
\end{aligned}
$$

(Aki and Richards 1980), where $\hat{\mathbf{x}}_{1}, \hat{\mathbf{x}}_{2}$, and $\hat{\mathbf{x}}_{3}$ are unit vectors towards north, east, and down. Expressed by fault parameters, the moment tensor function $\mathbf{M}(t)$ of Eq. (3) for a tensile fault segment in terms of the three directions of north, east, and down is:

$$
\begin{aligned}
M_{11}(t) & =\mu A\left[\left(\lambda / \mu+2 \sin ^{2} \delta \sin ^{2} \phi_{s}\right) \Delta u_{(n)}(t)\right. \\
& \left.-\left(\sin \delta \cos \lambda_{s} \sin 2 \phi_{s}+\sin 2 \delta \sin \lambda_{s} \sin ^{2} \phi_{s}\right) \Delta u_{(f)}(t)\right] \\
M_{22}(t) & =\mu A\left[\left(\lambda / \mu+2 \sin ^{2} \delta \cos ^{2} \phi_{s}\right) \Delta u_{(n)}(t)\right. \\
& \left.+\left(\sin \delta \cos \lambda_{s} \sin 2 \phi_{s}-\sin 2 \delta \sin \lambda_{s} \cos ^{2} \phi_{s}\right) \Delta u_{(f)}(t)\right] \\
M_{33}(t) & =\mu A\left[\left(\lambda / \mu+2 \cos ^{2} \delta\right) \Delta u_{(n)}(t)+\sin 2 \delta \sin \lambda_{s} \Delta u_{(f)}(t)\right] \\
M_{12}(t) & =\mu A\left[-\sin { }^{2} \delta \sin 2 \phi_{s} \Delta u_{(n)}(t)\right. \\
& \left.+\left(\sin \delta \cos \lambda_{s} \cos 2 \phi_{s}+0.5 \sin 2 \delta \sin \lambda_{s} \sin 2 \phi_{s}\right) \Delta u_{(f)}(t)\right] \\
M_{13}(t) & =\mu A\left[\sin 2 \delta \sin \phi_{s} \Delta u_{(n)}(t)\right. \\
& \left.-\left(\cos \delta \cos \lambda_{s} \cos \phi_{s}+\cos 2 \delta \sin \lambda_{s} \sin \phi_{s}\right) \Delta u_{(f)}(t)\right] \\
M_{23}(t) & =\mu A\left[-\sin 2 \delta \cos \phi_{s} \Delta u_{(n)}(t)\right. \\
& \left.-\left(\cos \delta \cos \lambda_{s} \sin \phi_{s}-\cos 2 \delta \sin \lambda_{s} \cos \phi_{s}\right) \Delta u_{(f)}(t)\right]
\end{aligned}
$$

For a limited fault segment, strike $\phi_{s}$, dip $\delta$, and slip $\lambda_{s}$ can be assumed to be constants. The tensile function $\Delta u_{(n)}(t)$ and the shear function $\Delta u_{(f)}(t)$ are two components of the displacement vector function $\Delta \boldsymbol{u}(t)$ of dislocation for a tensile fault segment. The Lamé constants, $\lambda$ and $\mu$, are two medium parameters near the fault segment. The ratio of $\lambda$ to $\mu$ in Eq. (9) can be expressed by Poisson's ratio $\sigma$ as: 
$\lambda / \mu=2 \sigma /(1-2 \sigma)$

In case of $\Delta u_{(n)}(t)=0$, Eq. (9) will be the same as Eq. (18) of Jost and Herrmann (1989), who assumed only the shear slip in the fault plane.

\section{3. $P$ AND $S$ WAVES IN HOMOGENEOUS MEDIA}

The energy is released partially as elastic waves out of a tensile fault segment when a jump of displacement occurs. The strengths of elastic waves radiating in a direction depend on the source couple with arm in the radiate direction. The forces of the couple may be oblique to its arm. Let the forces of the couple be decomposed into two components, the longitudinal component is a linear dipole with forces along the radiate direction and the transverse component is a shear couple with forces in the plane normal to the radiate direction. Such a linear dipole will contribute $P$ waves carrying the same strength and traveling in two opposite directions with a velocity of $\alpha$, whereas the shear couple contributes $S$ waves with a velocity of $\beta$. The displacements of $P$ and $S$ waves are proportional to the time derivatives of moment functions (Aki and Richards 1980; Pujol and Herrmann 1990) of the linear dipole and the shear couple, both are with arm in the radiate direction at the source.

Applying the tensor transformation, we know the source couple with arm in a radiate direction $\hat{\mathbf{r}}$ is $\mathbf{M} \hat{\mathbf{r}}$, where $\mathbf{M}$ is the moment tensor function of the tensile fault segment and $\hat{\mathbf{r}}$ is a unit column vector. The $P$ displacement, obtained by projecting $\mathbf{M} \hat{\mathbf{r}}$ in $\hat{\mathbf{r}}$ at the source, in a homogeneous medium is:

$\mathbf{u}^{P}=\frac{1}{4 \pi \rho \alpha^{3}} \frac{1}{r}\left[\hat{\mathbf{r}}^{\mathrm{T}} \dot{\mathbf{M}}(t-r / \alpha) \hat{\mathbf{r}}\right] \hat{\mathbf{r}}$

where $\rho$ is density, $r$ is radial distance, the super index $\mathbf{T}$ indicates a matrix transpose, the dot over $\mathbf{M}$ indicates derivative with respect to time. The $S$ displacement, obtained by subtracting the longitudinal component $\left(\hat{\mathbf{r}}^{\mathrm{T}} \mathbf{M} \hat{\mathbf{r}}\right) \hat{\mathbf{r}}$ from $\mathbf{M} \hat{\mathbf{r}}$ at the source, in a homogeneous medium is given by:

$$
\mathbf{u}^{S}=\frac{1}{4 \pi \rho \beta^{3}} \frac{1}{r}\left\{\dot{\mathbf{M}}(t-r / \beta) \hat{\mathbf{r}}-\left[\hat{\mathbf{r}}^{\mathrm{T}} \dot{\mathbf{M}}(t-r / \beta) \hat{\mathbf{r}}\right] \hat{\mathbf{r}}\right\}
$$

The $\mathbf{u}^{s}$ is orthogonal to $\hat{\mathbf{r}}$. Decomposing $\mathbf{u}^{s}$ into $S V$ and $S H$ displacements is easily achieved by introducing three orthogonal unit vectors, $\hat{\mathbf{r}}, \hat{\boldsymbol{\theta}}$, and $\hat{\boldsymbol{\varphi}}$ in spherical coordinates $(r$, $\theta, \phi)$ along a seismic ray radiated from the source shown in Fig. 1. $\theta$ is the incident angle measured from the downward vertical. $\phi$ is the azimuthal angle of the ray. $\hat{\boldsymbol{\theta}}$ and $\hat{\boldsymbol{\varphi}}$ are both transverse directions. The $S V$ and $S H$ displacements, obtained by projecting $\mathbf{M} \hat{\boldsymbol{r}}$ in $\hat{\boldsymbol{\theta}}$ and $\hat{\boldsymbol{\varphi}}$ at the source, in a homogeneous medium are given by:
$\mathbf{u}^{S V}=\frac{1}{4 \pi \rho \beta^{3}} \frac{1}{r}\left[\hat{\boldsymbol{\theta}}^{\mathrm{T}} \dot{\mathbf{M}}(t-r / \beta) \hat{\mathbf{r}}\right] \hat{\boldsymbol{\theta}}$

$\mathbf{u}^{S H}=\frac{1}{4 \pi \rho \beta^{3}} \frac{1}{r}\left[\hat{\boldsymbol{\varphi}}^{\mathbf{T}} \dot{\mathbf{M}}(t-r / \beta) \hat{\mathbf{r}}\right] \hat{\boldsymbol{\varphi}}$

where $\hat{\mathbf{r}}, \hat{\boldsymbol{\theta}}$, and $\hat{\boldsymbol{\varphi}}$ are three unit column vectors. In Cartesian coordinates of north, east, and down

$$
\begin{aligned}
& \hat{\mathbf{r}}=\sin \theta \cos \phi \hat{\mathbf{x}}_{1}+\sin \theta \sin \phi \hat{\mathbf{x}}_{2}+\cos \theta \hat{\mathbf{x}}_{3} \\
& \hat{\boldsymbol{\theta}}=\cos \theta \cos \phi \hat{\mathbf{x}}_{1}+\cos \theta \sin \phi \hat{\mathbf{x}}_{2}-\sin \theta \hat{\mathbf{x}}_{3} \\
& \hat{\boldsymbol{\varphi}}=-\sin \phi \hat{\mathbf{x}}_{1}+\cos \phi \hat{\mathbf{x}}_{2}
\end{aligned}
$$

$\mathbf{u}^{P}, \mathbf{u}^{S}, \mathbf{u}^{S V}$, and $\mathbf{u}^{S H}$ are the same as those expressions derived by Pujol and Herrmann (1990) for a generous body force representing a seismic source as a linear combination of couples. These are also presented for an arbitrary orientation of a double couple (Aki and Richards 1980). The elements of $\dot{\mathbf{M}}(t)$ are the time derivatives of $M_{l m}(t)$ in Eq. (9), where the tensile function $\Delta u_{(n)}(t)$ and the shear function $\Delta u_{(f)}(t)$ have taken account of time derivatives.

\section{RADIATION PATTERNS}

To investigate radiation patterns of $P$ and $S$ waves for a tensile fault segment dislocated in a direction, the displacement vector function is written as:

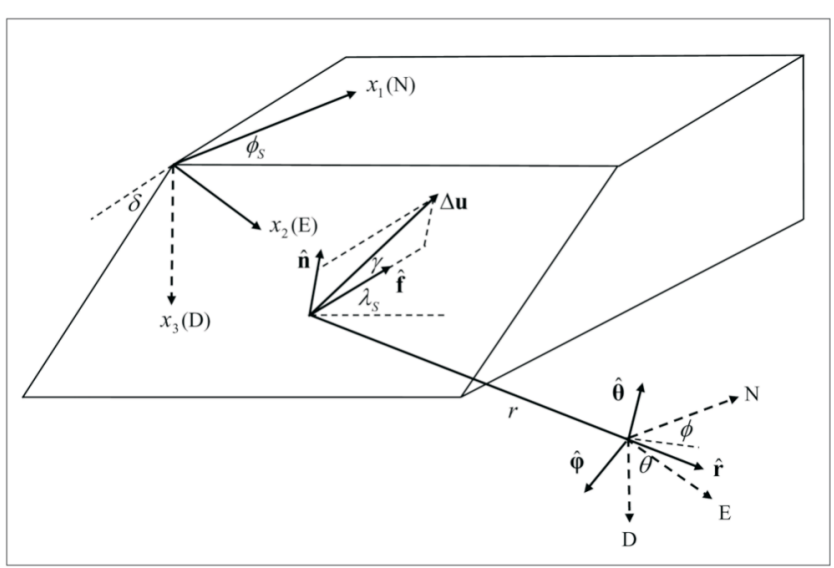

Fig. 1. Definition of a tensile fault segment and a seismic ray out of it in Cartesian coordinates of north (N), east (E), and down (D). The fault segment is defined by strike $\phi_{s}$ and $\operatorname{dip} \delta$. An arbitrary displacement vector of the movement of the hanging wall relative to the footwall is $\Delta \mathbf{u}$. $\hat{\mathbf{f}}$ is the unit slip vector in the fault plane. $\hat{\mathbf{n}}$ is the unit fault normal. $\lambda_{s}$ is the slip angle of $\hat{\mathbf{f}}$ measured from the direction of strike. $\gamma$ is the tensile angle measured from $\hat{\mathbf{f}}$ toward $\hat{\mathbf{n}}$. $\hat{\mathbf{r}}$ is the unit radial vector of the seismic ray. $r$ is radial distance. $\theta$ and $\phi$ are the incident angle and the azimuth of the ray. 


$$
\Delta \mathbf{u}(t)=\Delta u(t)(\sin \gamma \hat{\mathbf{n}}+\cos \gamma \hat{\mathbf{f}})
$$

where $\Delta u(t)$ is the magnitude of dislocation and $\gamma$ shown in Fig. 1 is the tensile angle measured from the slip vector $\hat{\mathbf{f}}$ in the positive sense as toward the fault normal $\hat{\mathbf{n}} . \gamma$ is positive if the fault segment expands, negative if it compresses. The $P, S V$, and $S H$ displacements for a tensile fault segment dislocated in a direction are written as:

$\mathbf{u}^{P}=\frac{\mu A \Delta \dot{u}(t-r / \alpha)}{4 \pi \rho \alpha^{3}} \frac{\left(\hat{\mathbf{r}}^{\mathrm{T}} \mathbf{S} \hat{\mathbf{r}}\right)}{r} \hat{\mathbf{r}}$

$\mathbf{u}^{S V}=\frac{\mu A \Delta \dot{u}(t-r / \beta)}{4 \pi \rho \beta^{3}} \frac{\left(\hat{\boldsymbol{\theta}}^{\mathrm{T}} \mathbf{S} \hat{\mathbf{r}}\right)}{r} \hat{\boldsymbol{\theta}}$

$\mathbf{u}^{S H}=\frac{\mu A \Delta \dot{u}(t-r / \beta)}{4 \pi \rho \beta^{3}} \frac{\left(\hat{\boldsymbol{\varphi}}^{\mathrm{T}} \mathbf{S} \hat{\mathbf{r}}\right)}{r} \hat{\boldsymbol{\varphi}}$

where the symmetric $\mathbf{S}$ is called the source dislocation tensor for a tensile fault segment dislocated in a direction. The elements of $\mathbf{S}$ in terms of the three directions of north, east, and down are:

$$
\begin{aligned}
S_{11} & =\left[2 \sigma /(1-2 \sigma)+2 \sin ^{2} \delta \sin ^{2} \phi_{s}\right] \sin \gamma \\
& -\left(\sin \delta \cos \lambda_{s} \sin 2 \phi_{s}+\sin 2 \delta \sin \lambda_{s} \sin ^{2} \phi_{s}\right) \cos \gamma
\end{aligned}
$$

$$
\begin{aligned}
S_{22} & =\left[2 \sigma /(1-2 \sigma)+2 \sin ^{2} \delta \cos ^{2} \phi_{s}\right] \sin \gamma \\
& +\left(\sin \delta \cos \lambda_{s} \sin 2 \phi_{s}-\sin 2 \delta \sin \lambda_{s} \cos ^{2} \phi_{s}\right) \cos \gamma
\end{aligned}
$$$$
S_{33}=\left[2 \sigma /(1-2 \sigma)+2 \cos ^{2} \delta\right] \sin \gamma+\sin 2 \delta \sin \lambda_{s} \cos \gamma
$$

$$
\begin{aligned}
S_{12} & =-\sin ^{2} \delta \sin 2 \phi_{s} \sin \gamma+\left(\sin \delta \cos \lambda_{s} \cos 2 \phi_{s}\right. \\
& \left.+\sin 2 \delta \sin \lambda_{s} \sin 2 \phi_{s} / 2\right) \cos \gamma
\end{aligned}
$$

$$
\begin{aligned}
S_{13} & =\sin 2 \delta \sin \phi_{s} \sin \gamma-\left(\cos \delta \cos \lambda_{s} \cos \phi_{s}\right. \\
& \left.+\cos 2 \delta \sin \lambda_{s} \sin \phi_{s}\right) \cos \gamma
\end{aligned}
$$

$$
\begin{aligned}
S_{23} & =-\sin 2 \delta \cos \phi_{s} \sin \gamma-\left(\cos \delta \cos \lambda_{s} \sin \phi_{s}\right. \\
& \left.-\cos 2 \delta \sin \lambda_{s} \cos \phi_{s}\right) \cos \gamma
\end{aligned}
$$

The source dislocation tensor for a tensile fault segment has five degrees of freedom of strike $\phi_{s}, \operatorname{dip} \delta, \operatorname{slip} \lambda_{s}$, tensile angle $\gamma$, and Poisson's ratio $\sigma$. Radiation patterns, which depend on the radiate direction $\hat{\mathbf{r}}(\theta, \phi)$, of $P$ and $S$ waves for a tensile fault segment $\left(\phi_{s}, \delta, \lambda_{s}, \gamma, \sigma\right)$ are:

$$
\Re^{P}=\hat{\mathbf{r}}^{\mathrm{T}} \mathbf{S} \hat{\mathbf{r}}
$$

$\Re^{S}=\left[\left(\hat{\boldsymbol{\theta}}^{\mathrm{T}} \mathbf{S} \hat{\mathbf{r}}\right)^{2}+\left(\hat{\boldsymbol{\varphi}}^{\mathrm{T}} \mathbf{S} \hat{\mathbf{r}}\right)^{2}\right]^{1 / 2}$

For an expansive fault segment shown in Fig. 2 with strike $0^{\circ}$, dip $90^{\circ}$, slip $0^{\circ}$, and Poisson's ratio 0.25 , the radiation patterns of the $P$ wave for tensile angles $\gamma$ of (a) $0^{\circ}$, (b) $10^{\circ}$, (c) $20^{\circ}$, and (d) $30^{\circ}$ are shown in Fig. 3. The $T$ and $P$ axes indicate the directions in which the maximum compression and dilatation waves are radiated. They are located on the plane containing the slip vector $\hat{\mathbf{f}}$ and the fault normal $\hat{\mathbf{n}}$. The $T$ axis will move toward $\hat{\mathbf{n}}$ and the $P$ axis is away from $\hat{\mathbf{n}}$ as the tensile angle increases. Both $T$ and $P$ rotate about the axis of $\hat{\mathbf{f}} \times \hat{\mathbf{n}}$ at the source through a half tensile angle, $\gamma / 2$, in the direction as a right-handed screw from those in the case of the shear faulting if Poisson's ratio is 0.25 . Amplitudes in the directions of $T, P$, and their vertical have $1,-1$, and 0 for the shear faulting in Fig. 3a to become 2, 0 , and 0.5 for a tensile angle of $30^{\circ}$ in Fig. $3 \mathrm{~d}$, where positive is for compression and negative for dilatation. The radiation pattern of the $P$ wave rotates about $\hat{\mathbf{f}} \times \hat{\mathbf{n}}$ at the source through an angle of $\gamma / 2$ for tensile faults. In the meanwhile, the amplitudes of the $P$ wave radiated on the plane normal to $\hat{\mathbf{f}} \times \hat{\mathbf{n}}$ superimpose an amount of $2 \sin \gamma$. However, the amplitude in the direction of $\hat{\mathbf{f}} \times \hat{\mathbf{n}}$ superimposes only an amount of $\sin \gamma$. Therefore, two nodal surfaces which separate the regions of compression and dilatation waves are

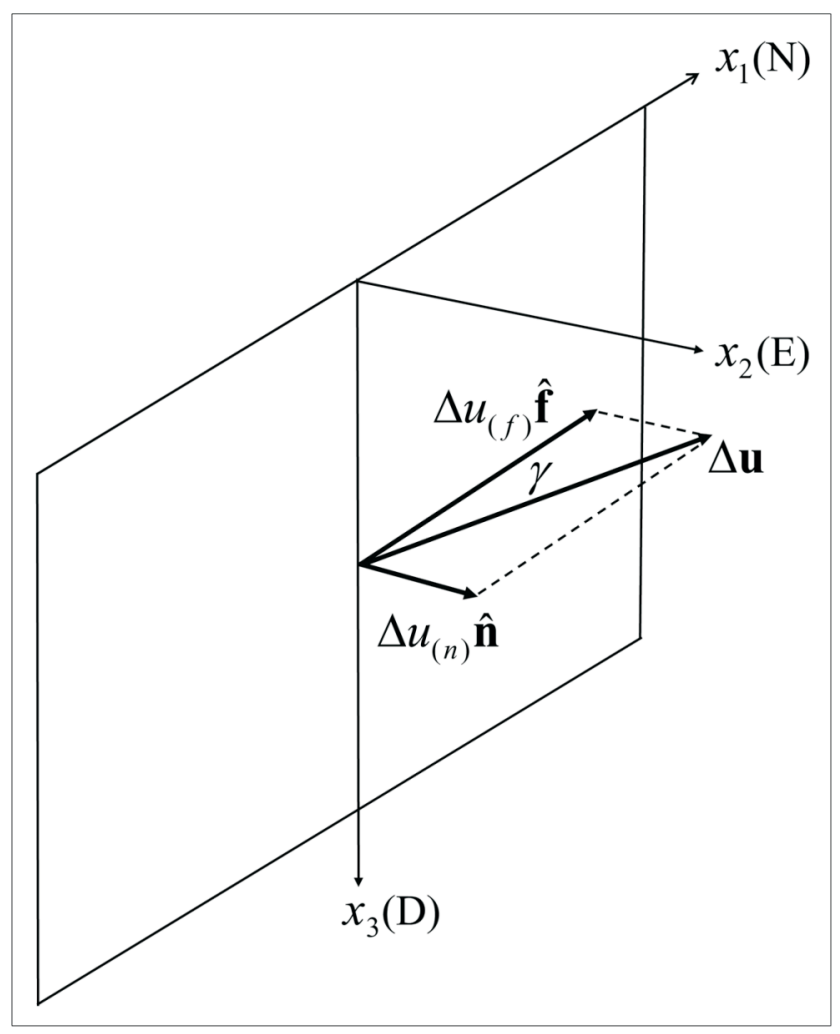

Fig. 2. An example of a tensile fault segment with strike $0^{\circ}$, dip $90^{\circ}$, slip $0^{\circ}$, and a tensile angle of $\gamma \cdot \Delta \mathbf{u}$ is the displacement vector. $\hat{\mathbf{f}}$ is the unit slip vector. $\hat{\mathbf{n}}$ is the unit fault normal. 

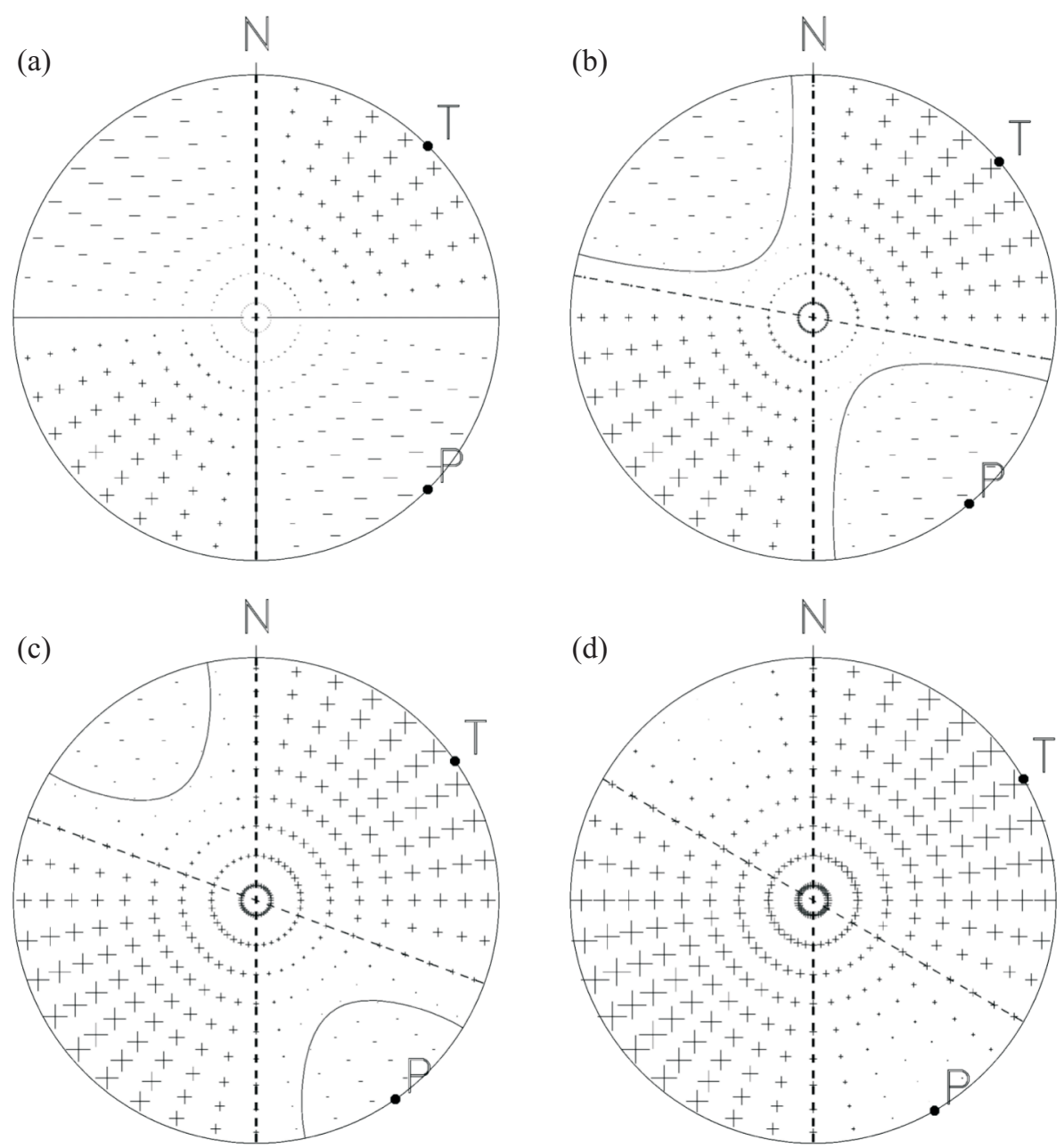

Fig. 3. Radiation patterns of the $P$ wave for an expansive fault segment in Fig. 2 with tensile angles of: (a) $0^{\circ}$, (b) $10^{\circ}$, (c) $20^{\circ}$, and (d) $30^{\circ}$. A lower focal hemisphere of the equal area projection is used. The primary and auxiliary fault planes are shown as dotted lines. The polarity and amplitudes are indicated by the scaled plus and minus signs. The nodal lines which indicate zero amplitude are plotted by sold lines. $T$ and $P$ are the tension and pressure axes.

no longer flat planes if the fault segment dislocates with a nonzero tensile angle. As a result, the compression region expands about the $T$ axis, whereas the dilatation regions shrink toward the $P$ axis for expansive faults. The span angle of each of the nodal surfaces is $\cos ^{-1}(2 \sin |\gamma|)$ in the plane normal to $\hat{\mathbf{f}} \times \hat{\mathbf{n}}$. Figures $3 \mathrm{~b}$ and $\mathrm{c}$ indicate that the nodal surfaces represented by solid lines are two cone-like surfaces, which are symmetric about the $P$ axis, one is open to northwest and another is to southeast. The two cone-like surfaces shrink to the $P$ axis and dilatation waves radiate nowhere as the tensile angle goes up to $30^{\circ}$ in Fig. $3 \mathrm{~d}$. In case of negative tensile angles, compressive faults, the $P$ axis will move toward $\hat{\mathbf{n}}$ as the $T$ axis is away from $\hat{\mathbf{n}}$. Two cone-like nodal surfaces will shrink toward the $T$ axis when the dilatation region expands about the $P$ axis. The radiation patterns of the $S$ wave for different positive tensile angles are shown in Fig. 4. The $T$ and $P$ are nodal axes, which will rotate about the axis of $\hat{\mathbf{f}} \times \hat{\mathbf{n}}$ at the source as the tensile angle increases, whereas the other nodal axis of $\hat{\mathbf{f}} \times \hat{\mathbf{n}}$ remains unchanged. The parti- cle motions of the $S$ wave are generally away from the $P$ axis and toward the $T$ axis. The radiation pattern of the $S$ wave on the plane containing the two nodal axes of $T$ and $P$ is invariant with tensile angle, but rotates about $\hat{\mathbf{f}} \times \hat{\mathbf{n}}$ at the source through an angle of $\gamma / 2$ in the sense of a right-handed screw. The amplitudes of the $S$ wave radiated on the plane containing the two nodal axes of $T$ and $\hat{\mathbf{f}} \times \hat{\mathbf{n}}$ will scale a factor of $1+\sin \gamma$ for tensile faults, whereas a factor of $1-\sin \gamma$ on the plane containing the two nodal axes of $P$ and $\hat{\mathbf{f}} \times \hat{\mathbf{n}}$. Therefore, more expansive faults will present stronger $S$ waves around the $T$ axis, and weaker $S$ waves around the $P$ axis. On the other hand, more compressive faults have stronger $S$ waves around the $P$ axis, and weaker $S$ waves around the $T$ axis.

\section{FOCAL MECHANISM}

To analyze the focal mechanism for a tensile fault seg$\operatorname{ment}\left(\phi_{s}, \delta, \lambda_{s}, \gamma, \sigma\right)$ dislocated in a direction, e.g., the dislo- 

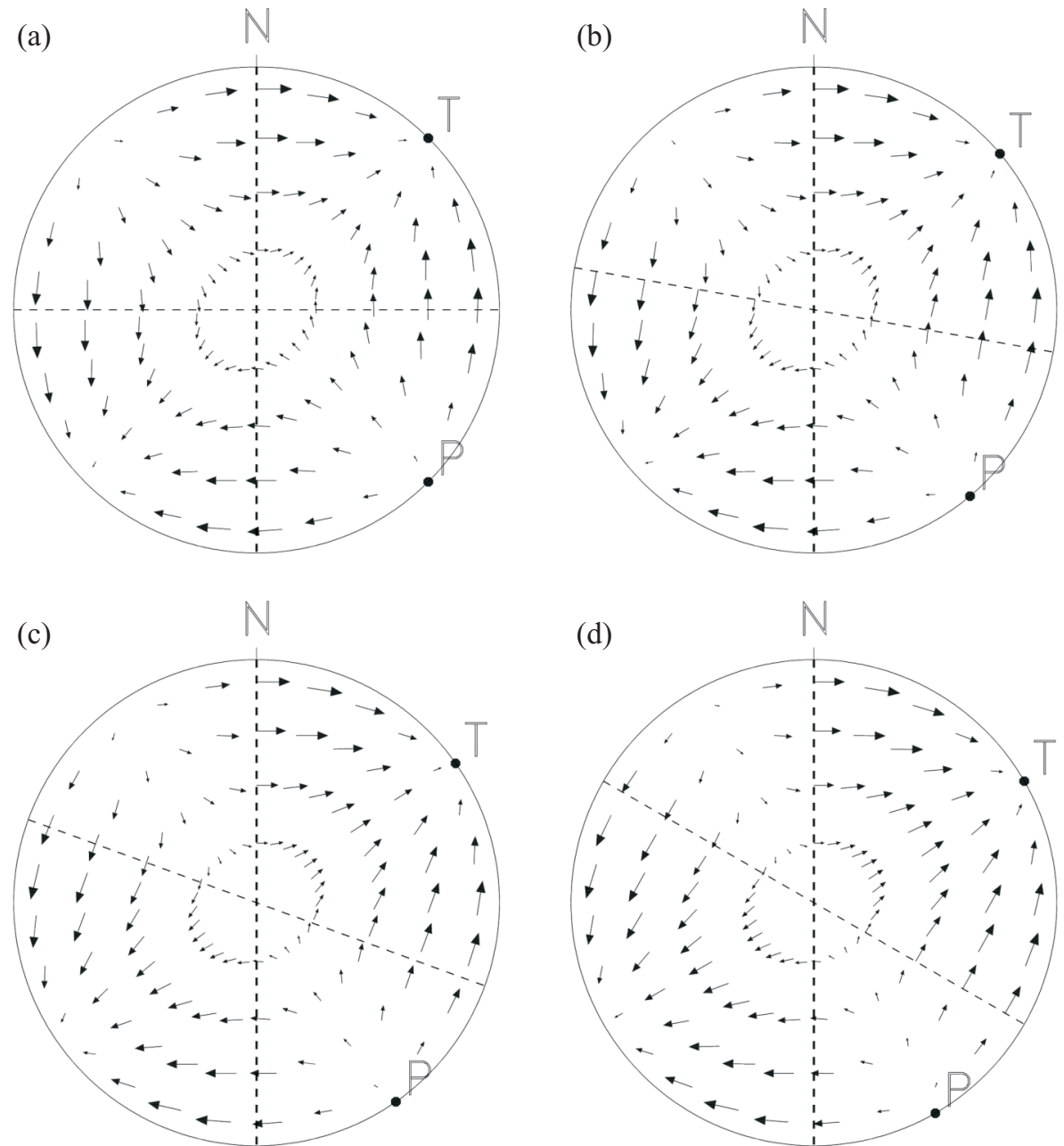

Fig. 4. Radiation patterns of the $S$ wave corresponding to the expansive faults in Fig. 3. The polarity and amplitudes are indicated by the scaled arrows which are projected in polarized planes tangent to the focal sphere.

cation for a focus at the origin time, we assume the displacement vector function $\Delta \mathbf{u}(t)$ of a step function: $\Delta u U\left(t-t_{0}\right)$. $\Delta u$ is the magnitude of dislocation. The unit step function $U(t-$ $\left.t_{0}\right)$ is equal to 1 when $t \geq t_{0}$ and 0 otherwise. This means that dislocation occurs for an instant in time at $t_{0}$. Then, the displacements of $\mathbf{u}^{P}$ and $\mathbf{u}^{S}$ at a radial distance $r$ will be proportional to two Dirac delta functions: $\delta\left(t-t_{0}-r / \alpha\right)$ and $\delta(t-$ $\left.t_{0}-r / \beta\right)$. The moment tensor of such a tensile fault segment is: $\mu A \Delta u \mathbf{S}$, where $\mathbf{S}$ is the source dislocation tensor. A scalar of $\mu A \Delta u$ can be ignored when we investigate the focal mechanism. The $\mathbf{S}$ is then thought of the moment tensor in which $\mu A \Delta u$ is equal to 1 .

The source dislocation tensor $\mathbf{S}$ of a tensile fault segment is real and symmetric, giving three eigenvalues and three orthogonal eigenvectors. In other words, any tensile fault segment can be equivalent to three orthogonal principal linear dipoles such that shear couples vanish. The maximum eigenvalue of the source dislocation tensor is the maximum tension of source to the external field. The direction of its corresponding eigenvector is called the tension axis in which the maximum compression wave is radiated. The minimum eigenvalue and the corresponding eigenvector indicate the maximum pressure and the pressure axis in which the maximum dilatation wave is radiated. The theory of the sum of eigenvalues gives the result that the trace of $\mathbf{S}$ :

$\operatorname{tr}(\mathbf{S})=S_{11}+S_{22}+S_{33}=[6 \sigma /(1-2 \sigma)+2] \sin \gamma$

which is invariant for any choice of three orthogonal coordinates and will be equal to the sum of three eigenvalues. The trace of $\mathbf{S}$ is always zero for a shear faulting, positive when a fault segment expands, and negative when it compresses.

If the source dislocation tensor $\mathbf{S}$ expressed in three directions of north, east, and down for a tensile fault segment has been inverted from the radiation pattern of $P$ or $S$ wave, then the focal mechanism can be analyzed, and the fault plane solution will be solved. First, the eigenvectors corresponding to the maximum and minimum eigenvalues of $\mathbf{S}$ 
are the tension and pressure axes. The tensile angle $\gamma$ of the fault segment can be found from the trace of $\mathbf{S}$ expressed by Eq. (25) if Poisson's ratio $\sigma$ is known. Then we form a source dislocation tensor $\mathbf{B}$ in terms of the three directions of the slip $\hat{\mathbf{f}}$, the fault normal $\hat{\mathbf{n}}$, and the $\hat{\mathbf{f}} \times \hat{\mathbf{n}}$ for the tensile fault segment. Summing up the source dislocate tensors in Eqs. (5) and (6) for the shear and tensile components of the displacement vector function in Eq. (18) and replacing $\lambda / \mu$ with a function of $\sigma$, Eq. (10), we obtain:

$$
\begin{aligned}
& \mathbf{B}= \\
& {\left[\begin{array}{ccc}
\{2 \sigma /(1-2 \sigma)\} \sin \gamma & \cos \gamma & 0 \\
\cos \gamma & \{2 \sigma /(1-2 \sigma)+2\} \sin \gamma & 0 \\
0 & 0 & \{2 \sigma /(1-2 \sigma)\} \sin \gamma
\end{array}\right]}
\end{aligned}
$$

where $B_{11}, B_{22}$, and $B_{33}$ indicate intensities of the linear dipoles along $\hat{\mathbf{f}}$, $\hat{\mathbf{n}}$, and $\hat{\mathbf{f}} \times \hat{\mathbf{n}}, B_{12}$ and $B_{21}$ are intensities of the double couples in the plane containing $\hat{\mathbf{f}}$ and $\hat{\mathbf{n}}$. $\mathbf{B}$ and $\mathbf{S}$ have the same eigenvalues. The eigenvectors of $\mathbf{B}$ corresponding to the maximum and minimum eigenvalues express the tension and pressure axes in the coordinate system of $\hat{\mathbf{f}}, \hat{\mathbf{n}}$, and $\hat{\mathbf{f}} \times \hat{\mathbf{n}}$. The transformation of an eigenvector $\mathbf{b}_{i}$ of $\mathbf{B}$ to an eigenvector $\mathbf{s}_{i}$ of $\mathbf{S}$ corresponding to the same eigenvalue $\lambda_{i}$ is:

$$
\mathbf{X b}_{i}=\mathbf{s}_{i}
$$

where $\mathbf{X}$ is an orthogonal matrix having the $\hat{\mathbf{f}}, \hat{\mathbf{n}}$, and $\hat{\mathbf{f}} \times \hat{\mathbf{n}}$ as the first, second, and third column vectors expressed in the coordinates of north, east, and down. If we form three eigenvectors of $\mathbf{B}$ and $\mathbf{S}$ in two matrices, $\mathbf{V}_{\mathbf{B}}$ and $\mathbf{V}_{\mathbf{S}}$, then Eq. (27) becomes:

$\mathbf{X} \mathbf{V}_{\mathbf{B}}=\mathbf{V}_{\mathrm{S}}$

where column vectors of $\mathbf{V}_{\mathbf{B}}$ and $\mathbf{V}_{\mathbf{S}}$ must be arranged in an order such that the same ordered ones correspond to the same eigenvalue. Let $\mathbf{V}_{\mathbf{B}}$ be an orthogonal matrix, then $\mathbf{X}$ can be solved as:

$$
\mathbf{X}=\mathbf{V}_{\mathbf{S}} \mathbf{V}_{\mathbf{B}}^{\mathbf{T}}
$$

There are eight solutions for $\mathbf{X}$ if we have three eigenvectors of $\mathbf{B}$ in two reverse directions and eigenvectors of $\mathbf{S}$ in fixed directions. Actually only four of $\mathbf{X}$ are independent. Two of independent $\mathbf{X}$ in which column vectors are right-handed provide the primary and auxiliary fault plane solutions. The first column vector of $\mathbf{X}$ indicates the slip direction $\hat{\mathbf{f}}$ in the fault plane. The second one is the fault normal $\hat{\mathbf{n}}$ which indicates a possible fault plane.

Eigenvalues of the source dislocation tensor $\mathbf{S}$ for any tensile fault segment are $\lambda_{1}=1+2 \sin \gamma, \lambda_{2}=\sin \gamma$, and $\lambda_{3}=$
$-1+2 \sin \gamma$ if Poisson's ratio is 0.25 , and the tensile angle $\gamma$ is $\sin ^{-1}[\operatorname{tr}(\mathbf{S}) / 5]$. The corresponding eigenvectors for the source dislocation tensor of the example in Fig. 2 in the coordinates of north, east, and down are:

$\mathbf{s}_{1}=\left[\begin{array}{c}\sqrt{(1-\sin \gamma) / 2} \\ \sqrt{(1+\sin \gamma) / 2} \\ 0\end{array}\right], \quad \mathbf{s}_{2}=\left[\begin{array}{l}0 \\ 0 \\ 1\end{array}\right], \quad \mathbf{s}_{3}=\left[\begin{array}{c}-\sqrt{(1+\sin \gamma) / 2} \\ \sqrt{(1-\sin \gamma) / 2} \\ 0\end{array}\right]$

The tension and pressure axes are $T$ and $P$ in Fig. 3. Both axes are always orthogonal. They are located at the symmetric axes of compression and dilatation regions, and agree with directions of the maximum compression and dilatation waves radiated. Two dotted lines indicate the primary and auxiliary fault planes. The heavy one is consistent with the fault plane assumed in the example. The poles of the primary and auxiliary fault planes are located in the plane containing the $T$ and $P$ axes. In the cases of tensile fault, the primary and auxiliary fault planes are no longer mutually perpendicular, and not consistent with the nodal surfaces of the $P$ wave. The span angle $\psi$ of the nodal surface in the plane containing the $T$ and $P$ axes depends on the tensile angle $\gamma$. The $|\gamma|$ is equal to $\sin ^{-1}(0.5 \cos \psi)$. $\gamma$ is positive if the compression region expands about the $T$ axis and negative if the dilatation region expands about the $P$ axis. The primary and auxiliary fault planes deviate at an angle of $45^{\circ}+\gamma / 2$ from the $T$ axis and an angle of $45^{\circ}-\gamma / 2$ from the $P$ axis, and have the nodal surfaces of the $P$ wave equally in between them. Both planes intersect with angles of $90^{\circ} \pm \gamma$ for tensile faults. Shown in Fig. 4, the particle motions of the $S$ wave are generally perpendicular to the primary and auxiliary fault planes at the source.

\section{DISCUSSION}

The seismic radiation from a tensile fault segment is a linear combination of those from the shear faulting and the tensile motion. The two components might have quite different types of functions in time. The shear faulting always goes forward in a direction then stops. It is usually represented by a ramp function characterized by a final slip. The tensile motion would move fault walls compactly, or move them apart then back together. If the tensile motion shapes like a ramp function, it will keep the spectral shape of seismic waves unchanged and affect only the spectral level. If the tensile function shapes like a pulse and ends without net displacement, the ground at far field due to the tensile motion will oscillate like a sinusoidal cycle such that the spectrum of ground displacement peaks at a certain frequency and decays with frequency on either side of the peak. Therefore, tensile faults ending without net volume change will enhance seismic waves at some frequencies, but not the seismic moment. 
To understand the phenomenon of volume dilatancy within and nearby a fault zone prior to a tremendous earthquake, the crust deformations observed on the Earth surface have been investigated. We can evidence the volume dilatancy by calculating the magnitude of compression on the fault from the radiation pattern of first arrivals of a tremendous earthquake. The compression reflects how far the fault walls have been moved apart to accommodate the slip of the earthquake. On the other hand, if we obtain the magnitude of compression during a foreshock, the dilatancy nearby can be inferred. This provides a new possibility for earthquake prediction. Besides the radiation pattern of first arrivals, the full waveforms from seismic stations could provide us with a window into the tensile function of a fault segment during its dislocation. By learning how expansion and compression influence the whole fault plane, we will better understand the tensile effect's impact on inducing and affecting the development of an earthquake.

Acknowledgements This work has benefited greatly from the Computer Programs in Seismology (VIII) provided by Robert B. Herrmann. I also thank two anonymous reviewers for suggesting improvements.

\section{REFERENCES}

Aki, K. and P. Richards, 1980: Quantitative seismology: Theory and methods. W. H. Freeman and Company, 932 pp.

Anooshehpoor, R. and J. N. Brune, 1992: Normal vibration, fault separation, and heat generation during stick-slip events in foam rubber. Seismol. Res. Lett., 63, 75.

Anooshehpoor, R. and J. N. Brune, 1994: Frictional heat generation and seismic radiation in a foam rubber model of earthquake. Pure Appl. Geophys., 142, 735-747.

Ben-Menahem, A. and S. J. Singh, 1981: Seismic waves and sources. Springer-Verlag New York Inc. 1108 pp.

Burridge, R. and L. Knopoff, 1964: Body force equivalents for seismic dislocations. Bull. Seismol. Soc. Am., 54, 18751888.

Dreger, D. S., H. Tkalčić, and M. Johnston, 2000: Dilatational processes accompanying earthquakes in the Long Valley Caldera. Science, 288, 122-125.

Dziewonski, A. M., G. Ekström, J. E. Franzen, and J. H. Woodhouse, 1987: Centroid-moment tensor solutions for January-March 1986. Phys. Earth Planet. Inter, 45, 1-10.

Frohlich, C., 1994: Earthquakes with non-double-couple mechanisms. Science, 264, 804-809.

Gilbert, F., 1970: Excitation of the normal modes of the Earth by earthquake sources. Gepphys. J. R. Astr. Soc., 22, 223-226.

Haskell, N. A., 1964: Total energy and energy spectral density of elastic wave radiation from propagating faults. Bull.
Seismol. Soc. Am., 54, 1811-1841.

He, Y. M., W. M. Wang, and Z. X. Yao, 2003: Static deformation due to shear and tensile faults in a layered half-space. Bull. Seismol. Soc. Am., 93, 2253-2263.

Herrmann, R. B., 1989: Seismic source inversion and earthquake ground motion modeling. Computer Programs in Seismology, VIII, Saint Louis University.

Hurukawa, N. and M. Imoto, 1993: A non double-couple earthquake in a subducting oceanic crust of the Philippine Sea plate. J. Phys. Earth, 41, 257-269.

Jost, M. L. and R. B. Herrmann, 1989: A student's guide to and review of moment tensors. Seismol. Res. Lett., 60, 37-57.

Julian, B. R., A. D. Miller, and G. R. Foulger, 1998: Non-double-couple earthquakes, 1, Theory. Rev. Geophys., 36, 525-549.

Kennett, B. I. N., 1983: Seismic wave propagation in stratified media, Cambridge University Press, 342 pp.

Knopoff, L. and M. J. Randall, 1970: The compensated linearvector dipole: A possible mechanism for deep earthquakes. J. Geophys. Res., 75, 4957-4963.

Kostrov, B. V. and S. Das, 1988: Principles of earthquake source mechanics, Cambridge University Press, $286 \mathrm{pp}$.

Lachenbruch, A. H. and J. H. Sass, 1980: Heat flow and energetics of the San Andreas fault zone. J. Geophys. Res., $\mathbf{8 5}, 6185-6222$.

Miller, A. D., G. R. Foulger, and B. R. Julian, 1998: Non-double-couple earthquakes, 2, Observations. Rev. Geophys., 36, 551-568.

Müller, G., 2001: Volume change of seismic sources from moment tensors. Bull. Seismol. Soc. Am., 91, 880-884.

Panza, G. F. and A. Sarao, 2000: Monitoring volcanic and geothermal areas by full seismic moment tensor inversion: Are non-double-couple components always artefacts of modeling? Geophys. J. Int., 143, 353-364.

Pujol, J. and R. B. Herrmann, 1990: A student's guide to point sources in homogeneous media. Seismol. Res. Lett., 61, 209-220.

Scholz, C. H., 1990: The Mechanics of Earthquakes and Faulting. Cambridge University Press, 439 pp.

Templeton, D. C. and D. S. Dreger, 2006: Non-double-couple earthquakes in the Long Valley volcanic region. Bull. Seismol. Soc. Am., 96, 69-79.

Xie, Y. S. and M. B. Cai, 1987: Compilation of historical materials of Chinese earthquakes. V3, Science Press, 1427 pp. (in Chinese)

Zheng, T. Y., Z. X. Yao, and P. C. Liu, 1995: The 14 November 1986 Taiwan earthquake - an event with isotropic component. Phys. Earth Planet. Inter, 91, 285-298.

Zoback, M. D., M. L. Zoback, J. P. Eaton, V. S. Mount, and J. Suppe, 1987: New evidence on the state of stress of San Andreas fault system. Science, 238, 1105-1111. 